\title{
Shaping Electron Bunches at the Femtosecond Level
}

\section{By crossing an electron beam with a terahertz light pulse, researchers are able to generate a tilted electron bunch, which could provide improved temporal resolution to electron microscopy.}

\section{by Jérôme Faure*}

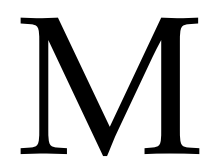
icroscopy is an extremely powerful tool for scientists. While biologists use light-based microscopes to observe cells, material scientists often rely on electrons, whose shorter wavelengths offer direct visualization of atoms with sub-angstrom resolution. Recently, the focus has been not only on resolving spatial features but also on obtaining temporal information that can reveal, for example, how atoms move in a crystal or how a molecule twists and turns during a chemical reaction [1]. To capture this behavior, ultrafast electron microscopy requires an electron bunch, or "flash," that is shorter than the timescale of the motion, which is typically in the femtosecond range. While femtosecond laser pulses have been around for more than three decades, it was not until recently that few-femtosecond electron bunches have become available $[2,3]$. The group of Peter Baum at Ludwig Maximilian University of Munich, which previously demonstrated alloptical control of electron bunches [4], has now gone one step further in the manipulation of electron bunch shape [5]. Using a terahertz $(\mathrm{THz})$ light pulse to alter the momentum of electrons in the beam, they obtain a femtosecond electron bunch with a "pulse front tilt," in which one side of the bunch travels ahead of the other side. Such tilted electron bunches could, in principle, further enhance the temporal resolution of electron microscopy.

A pulse front tilt occurs when there is a coupling between the spatial and temporal profile of a pulse such that the arrival time varies across the transverse position. The consequence of this geometry is that the energy front is not perpendicular to the propagation direction, as would normally be the case. In laser optics, a pulse front tilt can be obtained very easily by introducing angular dispersion-by using a prism, for example [6] (Fig. 1, top). As femtosecond light pulses have a broad spectral bandwidth, the different frequencies are deflected at different angles, resulting

* Laboratory of Applied Optics, ENSTA-CNRS-École Polytechnique, Palaiseau, France

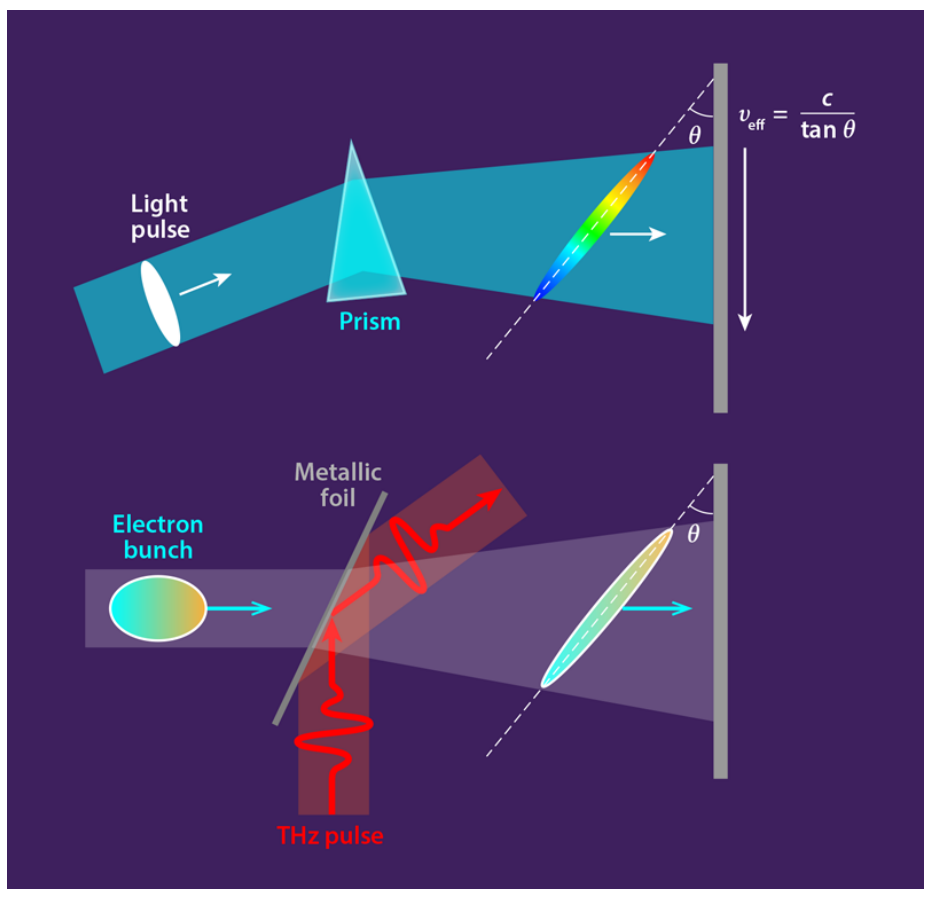

Figure 1: Illustration of a pulse front tilt in optics (top), in which a prism deflects light waves at different angles based on their frequencies. A new experiment has demonstrated a similar tilt for an electron bunch (bottom) by directing the electrons through a metallic foil at the same time as a THz pulse reflects off the foil. By adjusting the angle of the foil, researchers can control the amount of deflection and compression in the electron bunch. (APS/Alan Stonebraker)

in a pulse front tilt with a tilt angle $\theta$ determined by the prism's orientation. When such a tilted pulse strikes a plane perpendicular to the direction of propagation, energy gets deposited in a sequential fashion, like a "wave" of energy that starts at the top, say, and moves down the plane with an effective velocity $v_{\text {eff }}=c / \tan (\theta)$. Researchers exploit this tilted energy deposition for optical excitation of samples as well as for solving velocity matching issues in nonlinear optics [7]. 


\section{Physīcs}

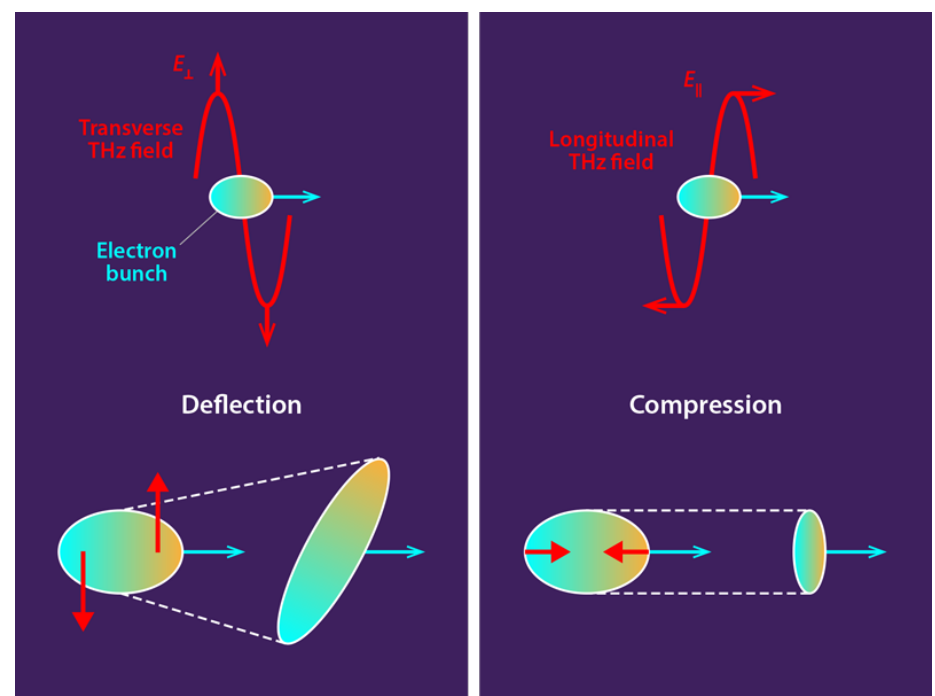

Figure 2: The two effects from the interaction of the $\mathrm{THz}$ pulse and electron bunch. When the pulse field is perpendicular to the bunch motion (left), the front and back of the bunch are deflected in opposite transverse directions. When the pulse field is parallel to the bunch motion (right), the bunch is compressed as the front is decelerated and the back is accelerated. (APS/Alan Stonebraker)

Generating a pulse front tilt with electrons is more challenging because electron bunches do not stay short as they propagate in vacuum: unlike photons, they repel each other, and their resulting velocity spread causes bunches to elongate. The Munich team's solution to this problem is to use intense $\mathrm{THz}$ light pulses, which are well adapted for manipulating electron bunches that are shorter than the $\mathrm{THz}$ wave period (roughly 1 picosecond). The experiment starts with a femtosecond laser pulse that is divided in two. The first part is sent into a photocathode in order to produce the ultrashort electron bunch. The second part of the pulse is sent into a nonlinear crystal that generates the $\mathrm{THz}$ pulse. The team arranges the pulse and bunch so that they cross paths at a thin metallic foil. This foil is a bit like a one-way mirror: it lets the electrons pass through but reflects the THz pulse, which means the bunch interacts with both the incoming light and the reflected light of the pulse (Fig. 1, bottom).

The pulse-bunch interaction is a combination of two separate effects that arise for different orientations of the $\mathrm{THz}$ pulse's electric field (Fig. 2). The first effect occurs when the electric field is perpendicular to the direction that the bunch propagates. Here, the electrons are transversally deflected with an angle that depends on their position in the bunch. As an example, the electrons in the front half of the bunch might be deflected to the left, while those in the back are deflected to the right. This deflection is called streaking because it normally creates a streak pattern on a detector. The second effect happens when the THz electric field is parallel to the electron bunch's direction. In this case, the faster- moving electrons in the front half are decelerated by the $\mathrm{THz}$ pulse, whereas the slower-moving electrons in the back are accelerated. After propagating a certain distance (to allow the back electrons to catch up to the front ones), the bunch length ends up being compressed.

The key to the Munich group's experiment is the foil and the angles it makes with respect to the $\mathrm{THz}$ pulse and the electron bunch. By adjusting these angles, the researchers are able to change the amplitudes of the parallel and perpendicular electric field components of the pulse and thereby control the pulse's effect on the electron bunch. For certain settings of the foil, the team obtains just the right amount of longitudinal compression and angular deflection so that the bunch emerges with a pulse front tilt. The maximum observed tilt angle is around $\theta=10^{\circ}$, which produces a picosecond delay between the leading edge of the bunch and the trailing edge.

This beautiful method could become particularly useful for following dynamics at the femtosecond timescale with so-called pump-probe microscopy. In this technique, a laser pulse excites a sample, while an electron bunch captures the dynamics at a specific time in the excitation cycle. Ideally, the pulse and the bunch strike the sample in such a way that they produce an "excitation wave" and a "probe wave" with the same effective velocity, $v_{\text {eff }}$, along the surface of the sample. In practice, this is very difficult to achieve, but pulse front tilting would give researchers an additional level of control for tuning the effective velocity at the sample. In a broader view, this work is evidence of the birth of femtosecond electron metrology and manipulation. I expect that more exotic electron pulses will become available, with more complex spatiotemporal correlations, potentially opening the door to a range of new applications in ultrafast probing.

This research is published in Physical Review Letters.

\section{REFERENCES}

[1] A. H. Zewail and J. M. Thomas, 4D Electron Microscopy (Imperial College Press, London, 2010).

[2] O. Lundh et al., "Few femtosecond, few kiloampere electron bunch produced by a laser-plasma accelerator," Nat. Phys. 7, 219 (2011).

[3] J. Maxson, D. Cesar, G. Calmasini, A. Ody, P. Musumeci, and D. Alesini, "Direct measurement of sub-10 fs relativistic electron beams with ultralow emittance," Phys. Rev. Lett. 118, 154802 (2017).

[4] C. Kealhofer, W. Schneider, D. Ehberger, A. Ryabov, F. Krausz, and P. Baum, "All-optical control and metrology of electron pulses," Science 352, 429 (2016).

[5] D. Ehberger, A. Ryabov, and P. Baum, "Tilted electron pulses," Phys. Rev. Lett. 121, 094801 (2018).

[6] M. R. Topp and Gail C. Orner, "Group dispersion effects in picosecond spectroscopy," Opt. Comm. 13, 276 (1975). 


\section{Physīcs}

[7] J. Hebling, G. Almasi, I. Kozma, and J. Kuhl, "Velocity matching

10.1103/Physics.11.87 by pulse front tilting for large area THz-pulse generation," Opt. Exp. 10, 1161 (2002). 